\title{
Une approche multiple de la catégorisation pour projet éthique
}

Éditorial

Stéphane Baele

\begin{abstract}
Dans cet essai introductif, nous partons d'un double constat pour souligner l'intérêt éthique de l'étude des catégories politiques. Ensuite, nous répertorions autour de deux pôles idéaux-typiques les grands axes d'analyse disponibles à qui souhaite intégrer le problème de la catégorisation dans une recherche de science sociale. Enfin, à la lumière de ces deux sections, nous présentons les différentes contributions.

Mots-clefs : catégorisation, éthique, interdisciplinaire

In this introductory essay, we start by putting forward a double statement over political categories, which allows us to underline the ethical ethos of categorisation studies. In a second step, we argue that these studies, which are more and more present in several scientific fields but always imply some kind of crossdiscipline perspective, gravitate around two ideal-types. Finally, we have a first glance at the following essays.
\end{abstract}

Keywords: categorisation, ethics, interdisciplinary

\section{Un double constat, une posture éthique}

En guise d'ouverture à ce numéro thématique sur la catégorisation politique, et avant d'en présenter les différentes contributions, il est nécessaire de revenir sur un double constat qui ne souffre pas contestation.

Le premier constat concerne une banalité dont la signification entière est pourtant d'une force peu commune : toute politique se base sur un jeu de catégories en relations. En d'autres termes, l'impact des catégories qui structurent nos lois et normes est potentiellement très grand - tout comme il peut également être, dans certains cas, négligeable. Il suffit de penser à quelques cas de politiques parmi les plus controversées de l'histoire récente pour se convaincre que le choix d'un jeu de catégories plutôt qu'un autre, lors de la lente et complexe émergence d'une régulation de n'importe quel type, peut avoir de graves répercussions. Ainsi, l'arrivée aujourd'hui particulièrement bien étudiée (par exemple CONTE \& ESSNER 1994 ; ESSNER 1995 ; PEGELOW 2006) des lois 
dites de Nuremberg, dans l'Allemagne nazie de 1935, illustre bien la construction de catégories susceptibles d'orienter - ou de cesser - la vie de certaines personnes. Plus récemment, mais sans portée analogique, on pourrait mettre en avant l'impact dramatique que peuvent entraîner les catégories de personnes et d'états qui structurent les politiques d'immigration occidentales. Ces deux exemples n'ont pas pour but de jeter la pierre aux catégories. Une telle posture, grotesque, ne peut émerger que si l'on méconnaît tout à la fois leurs rôles fondamental et social dans la perception. Ce qu'il faut donc voir, c'est l'inévitabilité de la catégorisation, véritable condition nécessaire à toute perception et action, mauvaise ou bonne. En revanche, comme nous allons le voir infra, rien n'indique que les contenus et limites des catégories que nous connaissons sont, aux aussi, incontestables. Pour le responsable politique autant que pour l'analyste, il importe de mieux connaitre ce mécanisme inévitable aux multiples dimensions et duquel peut émerger le pire autant que le meilleur.

C'est précisément cette nécessité d'investiguer les catégories qui est mise en doute lorsque - deuxième constat - s'entendent les fréquentes manifestations de lassitude visà-vis des différentes approches constructivistes en sciences sociales. Tant en sociologie qu'en épistémologie ou en science politique, une question revient avec insistance : «c'est construit, et alors ?». Autrement dit, si le message du (dé)constructivisme - à savoir, la réalité sociale est un construit, ou mieux, la réalité est un construit social - a bien été reçu, nombreux sont ceux qui pointent une impasse : à quoi servirait-il encore de déconstruire les catégories avec lesquelles nous avons prise sur le monde, si ce sont les seules que nous pouvons espérer avoir? Une telle critique est lourde d'une erreur et d'un sous-entendu de taille. L'erreur: croire que si la catégorisation est inévitable le contenu de ses produits - les catégories - l'est aussi. Le sous-entendu : nier, dans un réflexe réactionnaire, que mieux saisir un mécanisme central de notre compréhension et de notre action peut être vecteur d'un renouvellement positif de la pensée sociale et politique. À l'évidence, l'ensemble des contributions de ce numéro dénoncent ce sousentendu et réfutent cette erreur, directement ou indirectement.

La revue Émulations souhaite donc adresser ce double constat, de face. Vis-à-vis du premier, les diverses contributions montrent toutes que l'apparente banalité des catégories politiques résulte d'un enchevêtrement de processus psychosociaux plus ou moins conscients, plus ou moins stratégiques, et peut avoir d'importantes conséquences sur le plan éthique. Vis-à-vis du deuxième constat, le numéro prend le pari que mieux comprendre les différentes dynamiques en jeu lors de l'émergence, la pérennisation, et la disparition des catégories aide à poser les jalons d'une action sociale et politique de meilleure qualité, car simplement plus réflexive. À la question «c'est construit, et alors ? ", il ne s'agit pas tant de répondre par des critères d'utilité directe de la recherche, que par l'affirmation qu'une connaissance toujours plus fine des mécanismes en action lors de la constitution des catégories peut, peut-être, entraîner une plus grande prudence (au sens aristotélicien du terme) dans la conduite de nos projets. Ainsi, à 
l'instar des deux faces d'une même pièce, l'étude de la catégorisation est un mouvement éthique.

\section{Le défi d'une approche complexe de la catégorisation}

A l'évidence, la tâche est ardue. N'en déplaise à Chalmers (1995), qui liste la catégorisation parmi les «soft problems » amenant l'énigme suprême de la conscience, c'est-à-dire parmi ses questions non-inextricables (mais dont la solution nécessiterait tout de même encore «un ou deux siècles » d'investigations), la catégorisation est un processus intrinsèquement fuyant. Pour qui s'y essaye, l'étude des catégories présente en effet une complexité sans nom. Elle est d'abord le fruit d'un mouvement contradictoire de l'esprit, qui vise à comprendre ses propres règles de fonctionnement via la mise en application de ces règles. Mais au-delà de cet écueil épistémologique, la complexité de la question est aussi méthodologique, c'est-à-dire issue de sa centralité assumée ou non - dans de nombreux champs de recherche dont les langages et sens communs sont parfois dissonants. Intuitivement, on est donc tenté, pour voir clair, de subdiviser le champ des études de la catégorisation en accord avec les frontières disciplinaires. C'est d'ailleurs là une typologie d'autant plus pertinente qu'elle correspond à l'état de la recherche, tant l'imperméabilité des disciplines reste grande malgré les déclarations d'intention. D'un autre côté, force est de constater, au vu de la diversité des disciplines dont la catégorisation semble constituer le cœur des problèmes étudiés, que cette dernière aurait besoin d'être comprise par une théorie intégrant certains acquis d'origines diverses. Mais est-il seulement possible de faire dialoguer les acquis de théories aussi diverses - mais portant in fine sur la même chose - que l' « interpretive policy analysis », l'approche cognitive «theory-based categorisation », la sociologie de la connaissance scientifique, les généalogies foucaldiennes, la philosophie du deuxième Wittgenstein ou la pensée kantienne, les études sur la discrimination, la grammatologie derridienne et bien d'autres? Chacune a ses figures, ses procédures et méthodes, ses acquis et références, et pourtant il est évident qu'elles devraient montrer des compatibilités, peu importe la conception que l'on se fait de la science.

Un tel effort d'intégration peut potentiellement prendre trois formes. Si la multidisciplinarité est privilégiée, l'idée d'une théorie intégrée est écartée au profit d'une schématisation où dialogueraient les principaux acquis des différentes approches. Compatibilités et oppositions seraient soulignées dans ce qu'il conviendrait d'appeler l' «approche schématique ». Si le chemin est plutôt celui d'une véritable interdisciplinarité, il s'agirait pour le chercheur de forger de nouveaux concepts sur lesquels appuyer une approche originale en marge des enquêtes traditionnelles. Il faudrait alors parler d' "approche intégrée ». L'information constitue la dernière possibilité, consistant à partir d'un cadre théorique fourni au sein d'une discipline pour en repréciser certains éléments et en appuyer la force sur une série d'enseignements issus d'autres champs 
d'investigation. Le résultat serait une «approche informée ». À l'évidence, l'approche intégrée est trop complexe que pour être à l'ordre du jour. ${ }^{1}$ L'approche informée semble limitée mais peut s'avérer constituer une stratégie de recherche réellement intéressante. Ainsi par exemple, les travaux sociologiquement et épistémologiquement informés du psychologue Stephen Reicher (par exemple 2004, ou 2001) ou à l'inverse les études psychologiquement informées de l'anthropologue Dan Sperber (par exemple 1985) font preuve d'une réflexivité faisant évoluer notre compréhension de la catégorisation. En bout de course, c'est bien l'approche schématique qui apparaitt idoine, à mi-chemin entre d'une part les prétentions exagérées d'une théorie du tout, et d'autre part les portées limitées d'une stratégie de corrections.

\begin{tabular}{|l|c|c|c|}
\hline & Approche informée & Approche schématique & Approche intégrée \\
\hline Posture & Information & Multidisciplinarité & Interdisciplinarité réelle \\
\hline Pertinence & $\begin{array}{c}\text { Importante, mais } \\
\text { locale et stratégique }\end{array}$ & Importante & $\begin{array}{c}\text { Potentiellement cruciale, } \\
\text { mais au vu de l'ampleur de } \\
\text { la tâche, nulle. }\end{array}$ \\
\hline
\end{tabular}

En vue de préparer le terrain d'une telle approche schématique, il est donc sans doute plus pertinent de se figurer les grandes lignes de la recherche sur la catégorisation selon une typologie qui anticipe les différents recoupements et antagonismes des axes de recherche, que de perpétuer une typologie disciplinaire. À ce titre, une solution possible est d'imaginer un continuum entre deux idéaux-types d'études. D'un côté, on retrouverait une approche que l'on peut qualifier de «flexible » de la catégorisation, car elle présente ce processus comme étant vecteur de variabilité dans la perception et l'action. D'un autre côté se placerait une conception que l'on peut qualifier de "rigide », car elle insiste sur la stabilité des catégories. Entre ces deux conceptions se distribuent, en continuum, l'ensemble des contributions que l'anthropologie, la sociologie, la psychologie, la science politique et autres nous fournissent. Ce qui distinguent ces approches, ce n'est donc pas tant leurs dissensions quant à l'existence ou non de mécanismes de catégorisation plus ou moins largement partagés parmi les hommes, que la part qu'elles accordent dans ces mécanismes à la possibilité de variations dans les contenus des catégories. En effet, tant pour Kant que pour Wittgenstein, tant pour

1 Seuls Deleuze et Guattari ont un jour fait le pari d'une telle approche, et leur magistral diptyque Capitalisme et Schizophrénie a sans doute provoqué plus d'interrogations qu'il n'a permis aux sciences sociales de progresser. 
Aristote que pour les politologues de l'école «interprétative», il est évident que des processus nécessaires existent - autrement dit, que les catégories émergent selon un schéma dynamique plus ou moins constant. Ce qui diffère à chaque fois par contre, c'est la place laissée au contexte - à la contingence - dans lequel la catégorisation émerge. Ce qui distingue ou rapproche les contributions, c'est la mesure de l'impact contextuel.

Essayons à présent d'esquisser le paysage des contributions existantes sur la catégorisation qu'impliquerait cette nouvelle architecture. Classiquement, nous devrions commencer par associer la métaphysique d'Aristote à la tradition rigide : pour lui, il existe un nombre fixe (dix) de catégories immuables permettant l'organisation nécessaire et suffisante du monde. Ainsi de la «quantité », de la « relation », etc. À l'opposé se dresserait l'approche wittgensteinienne ${ }^{2}$, selon laquelle le sens d'un signifiant ne provient que de son insertion dans une pratique, toujours différente. Rien que de cette confrontation, que de nombreux chercheurs présentent comme cruciale, se dégage en fait un problème: en réalité, dans les théories «rigide» et «flexible» comprises de cette manière, la «catégorisation» ne recouvre pas la même chose. Dit simplement, les investigations d'Aristote ne portent pas sur la même chose que celles de Wittgenstein. D’un côté, la tradition rigide aristotélicienne s'occupe de définir la liste des catégories, entendues comme les classes les plus fondamentales d'objets. D'un autre côté, la tradition flexible wittgensteinienne insiste sur l'idée qu'une telle recherche de catégories fondamentales ne mène à rien, et qu'il faut plutôt investiguer les processus de catégorisation, qui ne peuvent manquer d'être empreints de contextualité.

On comprend dès lors qu'opposer Aristote et Wittgenstein sur la catégorisation ne veut pas dire grand chose. Il est plus intéressant de partir du cas ambigu présenté dans la Critique de la Raison Pure. Kant y reprend largement les catégories aristotéliciennes, mais pour leur faire jouer un rôle dans la dynamique de perception du monde. Les catégories, qui étaient ontologiques, se révèlent alors être des structures mentales, que l'on qualifierait aujourd'hui de cognitives. Dans cette optique, les catégories ne sont plus les classes fondamentales des objets, mais possèdent un rôle dynamique dans la perception et la construction du savoir. Même si toute une métaphysique contemporaine continue d'explorer les catégories ontologiques (par exemple CHISHOLM 1996 ; LOWE 1997 ; WesterhofF 2005), force est de constater que le terme de catégorie est depuis Kant subordonné à celui de catégorisation. À ce titre, la dette kantienne de la psychologie contemporaine est loin d'être reconnue et acquittée. Le problème n'est plus tant de savoir quelles seraient les classes les plus fondamentales des objets qui nous entourent, mais plutôt de comprendre comment les catégories que nous

\footnotetext{
${ }^{2}$ Nous considérons seulement ici l'approche du deuxième Wittgenstein.
} 
utilisons ont été construites, émergent de processus cognitifs ou sociaux. Ainsi, notre distinction entre théories «rigides» et «flexibles» n'oppose pas tant Aristote et Wittgenstein - la mise en avant de cette opposition classique avait valeur rhétorique que, par exemple, Kant et Wittgenstein. D'un côté, la catégorisation procède par des processus mentaux immuables qui informent les données du monde, et de l'autre la catégorisation procède par des processus mentaux et pratiques dont le fonctionnement lui-même est déterminé par un contexte toujours particulier. C'est ici qu'apparaissent vraiment les deux idéaux-types, dont Aristote est en fait exclu, autour de la question: quel est le poids du rôle du contexte dans la dynamique de catégorisation ? Pour Kant, la dynamique de catégorisation s'opère toujours de la même manière, peu importe le contexte. Pour Wittgenstein par contre, les éléments du contexte (au sens large) euxmêmes modifient la façon dont la catégorisation s'opère. En somme, la position actuelle de la recherche en sciences sociales vis-à-vis de la catégorisation peut se résumer selon le tableau suivant:

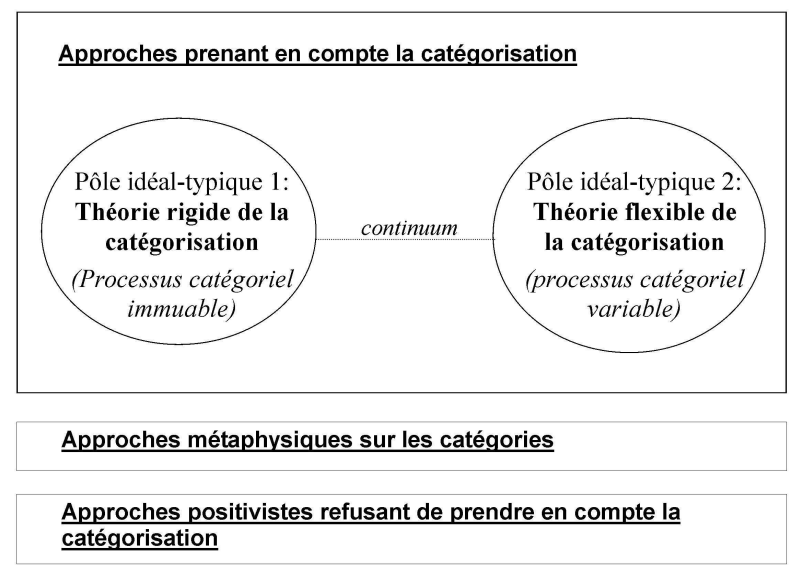

Près du pôle kantien, on retrouverait ainsi les approches dites «classiques » de la cognition, qui décrivent la catégorisation comme un processus de classement des phénomènes perçus selon leurs caractéristiques objectives communes. Les contenus catégoriels sont ainsi perçus comme largement nécessaires. De telles vues ne sont plus guère présentes dans la discussion en psychologie, qui privilégie aujourd'hui une vision plus wittgensteinienne de la catégorisation, où la diversité des catégories est soulignée. La psychologie cognitive actuelle présente en effet la catégorisation comme un processus de classement dont la logique n'est pas celle de critères objectifs nécessaires et suffisants, mais plutôt une logique de ressemblance voire de théorie (lire par exemple MEDIN 2005). Les critères pertinents n'y sont plus nécessaires, mais contingents, dépendants de croyances ("background knowledge»), de projets, d'interactions sociales. En sociologie et en science politique, les études de la catégorisation (qui restent 
quantitativement marginales) sont inévitablement des études à portée (dé)constructiviste, et donc flexibles. Pour ainsi dire, le simple projet de prendre en compte le problème de la catégorisation dans une étude l'oriente vers des prémisses (dé)constructivistes. À titre d'exemple, on peut mentionner la sociologie des sciences dite du «programme dur» (LATOUR \& WOOLGAR 1986, etc.), qui décrivent les dynamiques intersubjectives de création et de légitimation de catégories scientifiques. On peut aussi relever les efforts de l'« interpretive policy analysis », dont le but est de mettre à jour les processus sociaux par lesquels certaines catégories politiquement chargées émergent ou sont pérennisées (lire YANOW 1995 dans un numéro spécial de Policy Science), ou les études postcoloniales, qui se donne pour tâche - presque pour éthos - de déconstruire les catégories dominantes de lecture des inégalités Nord-Sud. On peut, enfin, souligner la force intacte que garde une approche foucaldienne en terme d'épistémè ou de gouvernementalité, qui constitue peut-être l'archétype de la théorie flexible. En anthropologie en revanche, l'état des lieux est moins clair, car si la qualité des approches comme celle de Medin et Atran (2004) - qui contestent l'existence de processus universaux de catégorisation - tirent vers l'idéal-type flexible, la discipline reste encore sous influence historique de courants globalisant soulignant le rôle de structures universelles de pensée, malgré l'importance du travail-clef de Lakoff (1987). Quant au panorama philosophique, il est évidemment éclaté. À titre d'exemple, l'approche searlienne de la catégorisation sociale apparait d'abord très flexible: les processus décrits (performativité, intersubjectivité, etc.) sont éminemment contextuels. Les catégories émergent d'actes et accords contingents, ne sont pas nécessaires. Cependant, deux nuances sont à apporter. D’abord, les processus de construction de la réalité sociale semblent bien être immuables, la catégorisation semble toujours émerger selon un même schéma peu variable. Ensuite, cette description flexible de la catégorisation sociale repose sur une conception en fait très rigide de la catégorisation des objets «bruts », qui sont sèchement distingués des objets et faits « sociaux ». Les catégories que nous utilisons en parlant de la nature - « une montagne », " de l'eau », etc. - n'apparaissent que comme les résultats d'une perception objective (perspective réaliste) qui ne pose pas question, qui n'est pas contestable, qui est celle du sens commun. L'approche searlienne de la catégorisation présente donc une combinaison d'éléments rigides et d'éléments flexibles. Quant aux autres approches philosophiques de la catégorisation, elles sont aussi variées qu'il existe de positions le long du continuum entre les idéaux-types rigide et flexible.

Un chercheur désirant prendre au sérieux le problème de la catégorisation dans une recherche de sciences sociales doit donc prendre acte de la tendance actuelle vers l'idéal-type flexible. Hormis en philosophie, dont le rôle est précisément de laisser se développer les thèses les plus divergentes sur ses problématiques, un consensus semble s'établir en faveur d'approches soulignant non seulement le caractère construit des catégories, mais, surtout, présentant la dynamique de catégorisation comme un processus combinant nécessité et contingence. Une approche schématique pourrait 
donc se construire, par exemple, autour de l'articulation entre une psychologie cognitive non-classique informée des travaux anthropologiques récents sur la catégorisation, une prise en compte wittgensteinienne des acteurs et des jeux de langage (voire des «speech acts» searliens) mais informée des acquis de l'«interpretive policy analysis », et une sociologie de la connaissance ou une épistémologie foucaldienne.

Voilà un programme pour le moins titanesque. Il est en réalité si chronophage et demandeur d'ouverture intellectuelle que l'on comprend aisément l'attrait plus raisonnable de l'approche informée ou, tout simplement, d'une recherche ignorant le problème de la catégorisation (cadre numéro 3 dans le schéma théorique supra). De même, on comprend tout autant la difficulté voire l'impossibilité de mener à terme une approche véritablement intégrée de la catégorisation.

\section{Contributions : de l'objet au sujet, de la pratique à la théorie}

C'est l'ampleur de cette tâche qui a poussé la revue Émulations à rassembler dans un même numéro différentes études de la catégorisation politique. En plus du présent essai, sept contributions offrent un panorama intéressant et de qualité sur la question. Si les thématiques abordées sont diverses, tous les articles participent en tout cas d'un même désir de disséquer les processus de catégorisation et d'en montrer les implications. Comment aborder ce numéro ? Deux logiques s'emboîtent. La première relève de ce qui est examiné : tandis que les premiers articles examinent des catégories d'objets, les suivants - majoritaires - se focalisent sur les catégories de personnes. Il nous semblait important non seulement de rappeler que la catégorisation politique est à la source d'objets a priori anodins ou du moins neutres, mais aussi que les dynamiques faisant émerger ces catégories-là sont largement les mêmes que celles qui conduisent aux catégories identitaires. La deuxième logique relève de la méthode. Tandis qu'un premier jeu de contributions s'intéresse à des catégories particulières, les deux dernières apportent un point de vue plus spécifiquement théorique.

Grégoire Lits ouvre ce numéro dans une perspective pragmatique, en questionnant de front le très actuel débat sur le nucléaire civil, à partir d'un examen de la Proposition de Directive européenne de novembre 2010 sur le traitement des déchets nucléaires. Ouvrant des pistes pour une généalogie des différentes catégories de déchets nucléaires, il montre que l'étude des catégories est aussi une analyse stratégique mettant en scène de multiples acteurs et savoirs en rapports de force. Dans un deuxième article de portée épistémologique aussi bien qu'éthique, Géraldine Thiry s'attache quant à elle à déconstruire un des indicateurs alternatifs au très critiqué PIB. Examinant les présupposés normatifs à la base de toute modélisation, son texte apporte un avertissement - derrière le chiffre se cache toujours la normativité - et un encouragement - tout indice n'est pas nécessairement mauvais, mais l'effort intellectuel 
pour sortir des schémas néfastes est tout à la fois violent et indispensable. Derrière ces deux contributions en résonance, l'article suivant opère le glissement entre catégories d'objets et de personnes. Par une étude sur les catégories de «sourd» et de "handicap», Pierre Schmitt montre toute la tension qui peut exister entre une définition "scientifique» d'une catégorie et une autre, produite par un mouvement social. Déjà est esquissée, dans ce texte, la question de l'identité. Cette question de l'identité, et cette tension naissant d'une définition problématique d'une catégoriegroupe, est prolongée dans l'étude de Germán Dario Fernandez. Celui-ci discute un cas de discrimination positive en Argentine, en mettant également l'accent sur la dimension pratique et vécue de la catégorisation. Enfin, Melaine Cervera et Renaud Hourcade proposent un surprenant jeu de miroir entre deux minorités pour qui l'enjeu est de rendre visible leurs catégories, dans une perspective de lutte sociale. En prenant l'exemple des noirs face au racisme et des sidéens face à la maladie, les auteurs montrent toute la dimension politique dans l'acte de se définir et de se montrer comme catégorie.

Ces différentes études, bien que solidement assises théoriquement, peuvent être reprises à la lumière des développements fournis par les deux dernières contributions aux accents nettement moins empiriques. ${ }^{3}$ Nicolas Mary présente une réflexion sur l'idée d'hors-groupe (en anglais : outgroup) en contournant originalement la psychologie sociale pour s'appuyer sur la sociologie de Merton. Une interview croisée menée par Stéphane Baele clôture le numéro, en mettant aux prises trois spécialistes reconnus de la catégorisation, sur les sept mêmes questions «naïves ». À ces questions déconnectées de tout cadre de référence théorique, et donc perturbantes, des points de vues que nous connaissons bien apparaissent alors en contraste. Amie Thomasson donne une lecture métaphysique, Dvora Yanow apporte celle de la théorie sociale, tandis que Thomas Serre propose des réponses empreintes de son travail en Intelligence artificielle. Nous remercions ces personnes pour le temps qu'elles ont consacré à cette entreprise, et déplorons cependant qu'à notre grand regret et malgré nos demandes répétées, notre interview croisée n’a pas suscité de réponses dans les temps de la part de psychologues reconnus.

Tant de choses peuvent être dites sur ces contributions, comme la prédominance des approches sociologiques par rapport aux études philosophiques ou psychologiques signe que le décloisonnement disciplinaire est loin d'être accompli - ou la prégnance de la question des minorités et des discriminations. Nous préférons en souligner ce qui

\footnotetext{
${ }^{3}$ Nous vous invitons également à consulter, sur notre site web, un essai de Nosetto, pleinement intégré à la logique de ce numéro thématique, qui rappelle toute l'importance de la pensée foucaldienne dans l'étude des catégories, celles-ci devant impérativement se comprendre dans des systèmes de savoir-pouvoir.
} 
apparait comme le point positif commun. Tous les textes, sans exception, ont mis en avant la dimension éthique du problème des catégories, même lorsque celles-ci apparaissent d'une objectivité scientifique. Plutôt que de la saisir dans toute sa splendeur théorique si complexe, plutôt que de l'observer en chercheurs passifs, la catégorisation est vue comme la clef des inégalités, mais également en même temps comme le lieu de construction possible d'une société juste. C'est en gardant sur soi cette possibilité que nous vous proposons de découvrir les multiples tiroirs de ce numéro thématique.

\section{Bibliographie introductive}

ARISTOTE (1963): Categories. Translated with notes by J.L. Ackrill. Oxford: Clarendon Press.

CONTE E., EsSNER C. (1994): «Völkerkunde et nazisme, ou l'ethnologie sous l'emprise des raciologues ». In L'Homme, vol.34 n¹29, pp.147-173.

ChALMERS D. (1995): «Facing up to the problem of consciousness». In Journal of Consciousness Studies, vol.3 nº 2 , pp.200-219.

Chisholm R. (1996) : A realistic theory of categories. Cambridge: Cambridge University Press.

ESSNER C. (1995): "Qui sera 'juif? La classification 'raciale' nazie, des lois de Nuremberg à la conférence de Wansee ». In Genèses, vol.21 n²1, pp.4-28.

KANT I. (2006): Critique de la raison pure. Paris: Flammarion.

LAKOFF G. (1987): Women, fire, and dangerous things. What categories reveal about the world. Chicago: University of Chicago Press.

Latour S, WoOlgar S. (1986): Laboratory Life. The social construction of scientific facts. Princeton: Princeton University Press.

LOWE J. (1997): «Ontological categories and natural kinds ». In Pbilosophical Studies, vol. $26 \mathrm{n}^{\circ} 1, \mathrm{pp} .29-46$.

MEDin D. (2005): « Concepts and conceptual structure ». In HAMILTON D., ed. (2004): Social cognition. Key readings in Social Psychology. New-York: Psychology Press, pp.115128.

Medin D., AtRAn S. (2004): "The native mind: Biological categorization and reasoning in development and across cultures ». In Psychological Review, vol.111 $\mathrm{n}^{\circ} 4$, pp.960-983. 
Pegelow T. (2006): «Determining 'People of German Blood', 'Jews' and 'Mischlinge': The Reich Kinship Office and the competing discourses and powers of Nazism, 1941-1943 ». In Contemporary European History, vol.15 n²1, pp.43-65.

REICHER S. (2004): «The context of social identity: Domination, resistance, and change ». In Political Psychology, vol.25 nº, pp.921-945.

REICHER S., HOPKINS N. (2001): « Psychology and the end of history: A critique and a proposal for the psychology of social categorization ». In Political Psychology, vol.22 $\mathrm{n}^{\circ} 2$, pp.383-407.

SEARLE J. (1995): The construction of social reality. New-York: Free Press.

SMiTH E., MEDIN D. (1981): Categories and Concepts. Cambridge MA: Harvard University Press.

SPERBER D. (1985): «Anthropology and psychology: Towards an epidemiology of representations ». In Man, vol.20 n¹, pp.73-89.

Westerhoff J. (2005): Ontological categories. Oxford: Clarendon Press.

WiTTGENSTEIN L. (2004): Recherches philosophiques. Paris: Gallimard - NRF.

YANOW D. (1995): « Editorial. Practices of policy interpretation». In Policy Sciences, n²8, pp.111-126. 